\title{
Effects of Short-Term Exercise in Overweight/Obese Adults with Insulin Resistance or Type 2 Diabetes: A Systematic Review of Randomized Controlled Trials
}

\author{
Tshidi Thaane ${ }^{1^{*}}$, Ayesha A Motala ${ }^{2}$ and Andrew J McKune ${ }^{1,3,4}$ \\ ${ }^{1}$ Discipline of Biokinetics, Exercise and Leisure Sciences, School of Health Sciences, University of KwaZulu-Natal, South Africa \\ ${ }^{2}$ Department of Diabetes and Endocrinology, School of Clinical Medicine, University of KwaZulu-Natal, South Africa \\ ${ }^{3}$ Discipline of Sport and Exercise Science, University of Canberra Research Institute for Sport and Exercise Science, Faculty of Health, University of Canberra, Australia \\ ${ }^{4}$ Collaborative Research in Bioactives and Biomarkers (CRIBB) Group, University of Canberra, Australia
}

${ }^{*}$ Corresponding author: Tshidi Thaane, Discipline of Biokinetics, Exercise and Leisure Sciences, School of Health Sciences, University of KwaZulu-Natal, 34 Somme Road, Musgrave, Durban, KwaZulu-Natal 400, South Africa, Tel: +27788871384; E-mail: t.thaane@gmail.com

Received date: November 21, 2018; Accepted date: December 20, 2018; Published date: December 31, 2018

Copyright: (C) 2018 Thaane T, et al. This is an open-access article distributed under the terms of the Creative Commons Attribution License, which permits unrestricted use, distribution, and reproduction in any medium, provided the original author and source are credited.

\begin{abstract}
Background: Chronic exercise training is associated with improvements in body composition and/aerobic fitness. This presents a challenge to study effects of exercise in isolation. Metabolic effects of short-term exercise training $(\leq$ 12 weeks) are unclear, with studies reporting improvements or no change in insulin sensitivity and/or glucose control. This review systemically examined randomized controlled trials (RCTs) to establish whether short-term exercise training improves insulin resistance and type 2 diabetes (diabetes).

Methods: Following the PRISMA guidelines, a systematic review was conducted on nine electronic databases (BMC Endocrine Disorders, Clinical Key, Cochrane Library, EBSChost, PubMed, Scopus, Sabinet_SA Publications, The Lancet and Web of Science) to identify randomized controlled human trials (2005-2018) examining effects of short-term exercise training ( $\leq 12$ weeks) in overweight/obese adults with insulin resistance or diabetes. Search terms included: insulin resistance, type 2 diabetes, short-term, exercise or energy expenditure and randomized controlled trial. Studies were only included if they provided sufficient data on: insulin sensitivity, glycemic control, body composition and aerobic fitness.
\end{abstract}

Results: From 374 articles, three met the inclusion criteria. Of these, two prescribed moderate intensity training (MIT); rate of perceived exertion (RPE) $12-13$ and $60 \%$ lactic threshold (LT) for four and twelve weeks, respectively while one prescribed vigorous exercise training at $70 \%$ VO2max for seven days. Duration of exercise sessions was 40-60 minutes. Twelve weeks of MIT was associated with improved glycemic control vs. no change in the 7-d and four weeks studies. Seven days of vigorous training was associated with greater improvement in insulin sensitivity, $44.4 \%$ vs. no change in the four- and twelve-weeks studies.

Conclusion: Short-term exercise appears to improve insulin sensitivity and glucose control independent of body fat loss or gains in aerobic fitness. Vigorous exercise training was associated with superior improvements in insulin sensitivity. More RCTs are needed to confirm these findings.

Keywords: Metabolic stress; Peripheral vascular disease; Physical activity

\section{Introduction}

Insulin maintains glucose homeostasis by promoting gluconeogenesis and glycogenesis [1]. A defect in the metabolic insulin-signalling pathway therefore, leads to disorders including peripheral vascular disease (PVD) and type 2 diabetes mellitus (diabetes) [2]. The pathogenesis of insulin resistance is unclear, however, the disorder has been reported to be a precursor for diabetes which has reached epidemic proportions and shows no sign of abatement [3]. Diabetes is characterized by hyperglycemia that results from the inadequate islet beta $(\beta)$ cell and adipose-tissue responses to chronic fuel excess which results in metabolic stress that causes macroand microvascular complications including nephrology, retinopathy and neuropathy [4].
Emerging evidence indicates that susceptibility to diabetes may be acquired early in life due to foetal and neonatal programming that occurs via epigenetics phenomena, suggesting that interventions to improve maternal and early child health may be crucial for diabetes prevention. In adults, intensive lifestyle modification (ILM); diet and exercise, is recommended as prevention and management strategy for insulin resistance and diabetes [5]. ILM has been reported to offer long-term clinical benefits such as improvement of insulin sensitivity, glucose control, body composition and cardiorespiratory fitness [6-8]. Diabetes patients are encouraged to accumulate at least 150 minutes of physical activity per week, with more hours increasing benefit [9]. Previous reviews and meta-analyses have reported that exercise training improves glycaemic control in patients with insulin resistance or diabetes [10]. In these reviews, however, both short-term and chronic exercise training interventions are included [11]. In chronic studies, exercise-induced improvements in insulin resistance or 
diabetes are generally accompanied by improvements in body composition and aerobic fitness. This presents a challenge to study the effects of exercise in isolation. Thus, short-term exercise interventions are increasingly being investigated to better understand independent effects of exercise on insulin resistance and diabetes. Findings from available short-term studies vary; some studies have reported that exercise improves in insulin sensitivity and glucose control while others have reported modest or no change [12,13].

The current review therefore, systemically examined randomized controlled trials with insulin resistance or diabetes patients to determine whether short-term exercise training of 12 weeks or less improves insulin resistance and diabetes in overweight/obese adults.

\section{Methods}

\section{Search strategy}

This review was conducted in accordance with the Preferred Reporting for Systematic Reviews and Meta-Analyses (PRISMA) guidelines [9]. Details of the protocol for this systematic review were registered on PROSPERO and can be accessed at http:// www.crd.york.ac.uk/PROSPERO/display_record.php?

$I D=C R D 42018086777$ Electronic searches were conducted in nine databases; BMC Endocrine Disorders, Clinical Key, Cochrane Library, EBSChost, PubMed, Scopus, Sabinet_SA Publications, The Lancet and Web of Science for prospective studies published from 2005 to 2018. The database searching was conducted using the keywords; insulin resistance, type 2 diabetes, exercise, aerobic training, overweight, obesity, adults, randomized controlled trial, energy expenditure, impaired glucose tolerance. The search was restricted to peer reviewed original research with full-text published in English. Full-text of articles relevant to the current review was available. The reference list of key articles was manually searched for potentially eligible studies to ensure that all trials relevant to this review had been identified.

\section{Search terminology}

Medical subject heading used in the current review included: insulin resistance OR type 2 diabetes OR obesity AND exercise OR energy expenditure OR aerobic fitness AND glucose control OR lipid profile.

\section{Inclusion and exclusion criteria}

Studies were considered for inclusion if they met the following criteria: 1) Overweight/obese study participants with clearly defined insulin resistance or diabetes status and without co-morbidity; 2) exercise training of any mode, frequency, duration and intensity but follow-up period of twelve weeks or less; 3 ) studies must have reported on pre and post measures of at least one marker of insulin sensitivity or glucose control, body composition or aerobic fitness; 4) studies must have included details of the outcome measure including instrument, and method as well as methods of data collection and analysis; 5) studies must have randomly assigned participants to exercise and no exercise control groups. Studies with combined exercise and dietary interventions were only considered for inclusion if dietary intervention was uniform across all groups. Review articles and/meta-analysis, author manuscripts and letters to editors were excluded.

\section{Outcome measures}

The primary outcomes of the current review included markers of insulin resistance and glucose control (HOMA-IR, QUICKI, HbAlc, FPG), anthropometry (body mass index, body fat) and aerobic fitness $\left(\mathrm{VO}_{2} \mathrm{max}, \mathrm{VO}_{2}\right.$ peak). Data in the studies included in the review were expressed as mean and standard deviation/standard error of the mean and percentage change. The selected outcomes were chosen because they are interrelated. Insulin resistance contributes to impaired glucose control which can cause hyperlipidemia and systemic inflammation. Improvements in body fat and aerobic fitness have been found to improve insulin sensitivity [10-12].

\section{Data extraction}

A standardized pre-piloted data extraction form [Joanne Briggs Institute (JBI) Data Extraction Form for Experimental/Observational Studies] was used to extract data from the included studies [13]. Extracted information included study method, study setting and population, sample size, details of the intervention (exercise mode, frequency and duration as well as duration of the intervention period) and measured outcomes.

\section{Study quality assessment}

The JBI Critical Appraisal Checklist for Randomized Controlled Trials was used to assess the methodological quality and to determine the extent to which a study addressed the possibility of bias in its design, conduct and analysis [13]. The tool consists of 13 items rated as 'no, 0; unclear, 0; and yes, 1' and includes criteria such as: clear description of the aims, interventions, outcome measurements and participants, representativeness of participant groups, appropriateness of statistical analyses. The checklist included the following questions: 1) was true randomization used; 2) were participants and/ assessors blinded to assignment; 3 ) were participants analysed in the groups to which they were randomized. The outcome of the critical appraisal was used to inform synthesis and interpretation of the results.

\section{Data analysis}

A narrative synthesis of the results was conducted due to the heterogeneity in the measurements of insulin sensitivity and follow-up period of studies included within this review. An increase in the output from research on short-term exercise in insulin resistant or type 2 diabetes will allow for meta-analysis to be conducted.

\section{Results}

\section{Identification and selection of studies}

Electronic searches yielded 374 articles while manual search yielded two. When duplicates and studies that did not meet the inclusion criteria were removed, three studies remained for quantitative analysis. The most common reasons for exclusion were; topic not relevant to the current review, study not a randomized controlled trial, insulin resistance or diabetes status not clear and intervention longer than twelve weeks (Figure 1). 
Citation: Thaane T, Motala AA, McKune AJ (2018) Effects of Short-Term Exercise in Overweight/Obese Adults with Insulin Resistance or Type 2 Diabetes: A Systematic Review of Randomized Controlled Trials. J Diabetes Metab 9: 816. doi:10.4172/2155-6156.1000816

Page 3 of 5

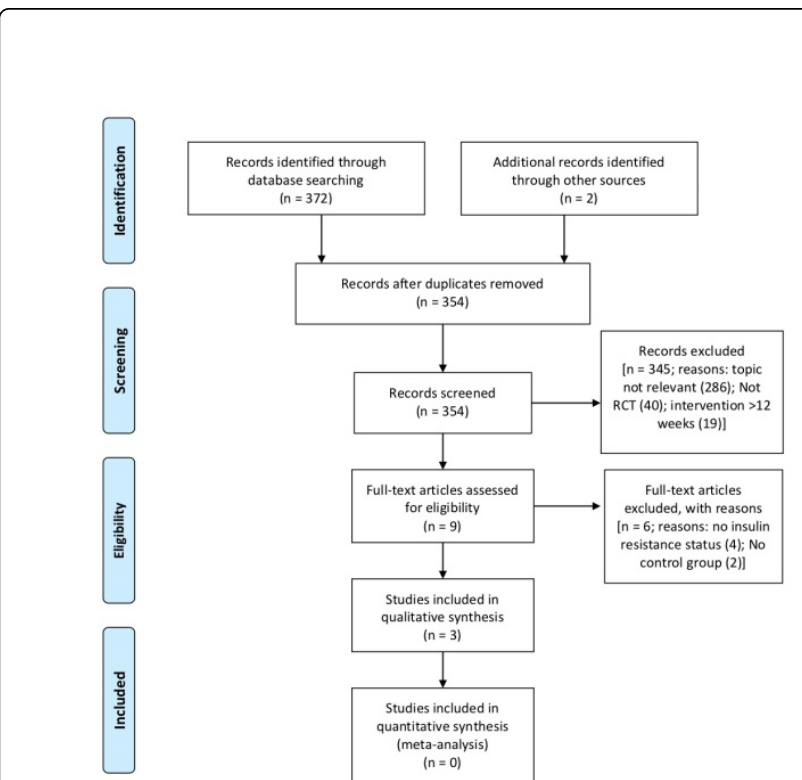

Figure 1: PRISMA Flow chart. RCT; Randomized controlled trial.

\section{Study characteristics}

Trial settings and participants: Table 1 shows the characteristics of studies included in the review. Exercise programme settings included University and University hospital $[14,15]$ and clinical research centres [16]. The total number of participants in the included trials ranged from 18 to 132. The mean age ranged from 48 and 65 years.

Intervention: Studies included in this review prescribed individualized aerobic and/or resistance exercise. Of the three studies, one study prescribed aerobic training combined with a balanced (BAL) diet which consisted of $50 \%$ carbohydrates, $30 \%$ fat and $20 \%$ protein [16]. Two studies prescribed moderate intensity exercise while the remaining study prescribed vigorous intensity exercise.

The mode of aerobic exercise used included walking, jogging and running. Progressive resistance training used light weights with exercises designed to target major muscle groups such as legs, shoulders and back.

The duration of exercise sessions in all three trials was 40-60 minutes. Combined aerobic and exercise training groups performed the exercises at half the time required for aerobic and resistance training alone. Of the three trials, two studies prescribed 3 nonconsecutive exercise sessions per week for a duration of four and twelve weeks $[14,15]$. The remaining trial prescribed seven consecutive 50 minutes exercise sessions [16].

\begin{tabular}{|c|c|c|c|c|c|c|c|c|}
\hline \multirow[b]{2}{*}{$\begin{array}{l}\text { Sourc } \\
\text { e }\end{array}$} & \multirow[b]{2}{*}{$\begin{array}{l}\text { Sample size } \\
\text { (M/F) }\end{array}$} & \multirow[b]{2}{*}{$\begin{array}{l}\text { Age } \\
\text { (years) }\end{array}$} & \multirow[b]{2}{*}{$\begin{array}{l}\text { Study } \\
\text { quality }\end{array}$} & \multicolumn{3}{|c|}{ Intervention } & \multirow[t]{2}{*}{ Outcome measure } & \multirow{2}{*}{ Findings } \\
\hline & & & & Group & Intensity & $\begin{array}{l}\text { Frequency } \\
\text { and } \\
\text { duration }\end{array}$ & & \\
\hline \multirow{4}{*}{ [15] } & $12(5 / 7)$ & 52.09 & Moderate & AET & \multirow{4}{*}{ LTHR } & \multirow{4}{*}{$\begin{array}{l}3 \mathrm{~d} / \text { week-12 } \\
\text { weeks }\end{array}$} & \multirow{4}{*}{$\begin{array}{l}\text { HOMA-IR, HbA1c, } \text { FPG, } \\
\text { PPG, TG, hs-CRP, BMI, } \\
\text { VO2peak }\end{array}$} & \multirow{4}{*}{$\begin{array}{l}\text { AET significantly improved fasting } \\
\text { glucose, TG and inflammation by } \\
15.6 \%, 11.2 \% \text { and } 10.8 \% \text {, RET lowered } \\
\text { these parameters by } 17.0 \%, 52.9 \% \text { and } \\
15.0 \% \text {, while AET+RET improved them } \\
\text { by } 9.0 \%, \quad 19.8 \% \text { and } 10.6 \% \\
\text { respectively. These improvements } \\
\text { occurred without changes in BMI and } \\
\text { WC in adults with type } 2 \text { diabetes }\end{array}$} \\
\hline & $12(5 / 7)$ & 54.1 & & RET & & & & \\
\hline & $12(4 / 8)$ & 57.9 & & $\mathrm{AET}+\mathrm{RET}$ & & & & \\
\hline & $12(4 / 8)$ & 53.42 & & CNT & & & & \\
\hline \multirow[b]{2}{*}{ [14] } & $68(36 / 32)$ & 57 & Moderate & $\mathrm{AET}+\mathrm{RET}$ & \multirow[b]{2}{*}{ RPE 12-13 } & \multirow[b]{2}{*}{$\begin{array}{l}3 \mathrm{~d} / \text { week-4 } \\
\text { weeks }\end{array}$} & \multirow[b]{2}{*}{$\begin{array}{l}\text { HOMA-IR, HOMAß-cell, BG, } \\
\text { QUICKI, TG, BMI, VO2max }\end{array}$} & \multirow{2}{*}{$\begin{array}{l}\text { Combined AET and RET produced a } \\
\text { statistically significant } 0.2 \% \text { decrease in } \\
\text { TG with a small (1.4\%) increase in } \\
\text { VO2max but no change in BMI in } \\
\text { moderately healthy patients with type } 2 \\
\text { diabetes }\end{array}$} \\
\hline & $64(34 / 30)$ & 65 & & CNT & & & & \\
\hline \multirow{2}{*}{ [16] } & $9(6 / 3)$ & 48.4 & \multirow{2}{*}{ Moderate } & $\begin{array}{l}\mathrm{AET}+\mathrm{BAL} \\
\text { diet }\end{array}$ & \multirow{2}{*}{$\begin{array}{l}70 \% \\
\text { VO2max }\end{array}$} & \multirow{2}{*}{$7 d$} & \multirow{2}{*}{$\begin{array}{l}\text { WBIS, PIS, HIS, FPG, BF, } \\
\text { VO2max }\end{array}$} & \multirow{2}{*}{$\begin{array}{l}\text { AET + BAL diet significantly improved } \\
\text { WBIS and PIS by } 37.5 \% \text { and } 44.4 \% \text {, } \\
\text { respectively without changes in body fat } \\
\text { or aerobic fitness in obese adults with } \\
\text { mild type } 2 \text { diabetes }\end{array}$} \\
\hline & $9(7 / 2)$ & 50.9 & & BAL diet & & & & \\
\hline \multicolumn{9}{|c|}{$\begin{array}{l}\text { AET: Aerobic exercise training; RET: Resistance exercise training; LTHR: Lactate threshold heart rate; BAL: Balanced; CNT: Control; RPE: Rate of perceived exertion; } \\
\text { HOMA-IR: Homeostasis model assessment of insulin resistance; QUICKI: Quantitative insulin check index; WBIS: Whole body insulin sensitivity; PIS: Peripheral } \\
\text { insulin sensitivity; HIS: Hepatic insulin sensitivity; BG: Blood glucose; FPG: Fasting plasma glucose; PPG: Post prandial glucose; HbA1c: Hemoglobin A1c; TG: } \\
\text { Triacylglycerols; hs-CRP: High sensitivity C-reactive protein; BW: Body weight; BMI: Body mass index; WC: Waist circumference; BF: Body fat; SBP: Systolic blood } \\
\text { pressure, DBP: Diastolic blood pressure; VO2max: maximal aerobic capacity; VO2peak: Peak aerobic capacity. }\end{array}$} \\
\hline
\end{tabular}

Table 1: Characteristics of studies included in the review (n:3). 


\section{Study quality}

Overall the studies included in this review scored positively on the JBI Critical Appraisal Checklist for Randomized Controlled Trials [13]. All the included studies treated groups identically other than the intervention of interest. Follow-up was complete, participants were analyzed in the to which they were randomized, and outcomes were measured in a reliable way.

\section{Study outcomes}

Insulin resistance: Three studies reported on markers of insulin resistance [14-16]. Of these, one study reported positive findings. Winnick et al reported that during a high-dose insulin postintervention clamp, exercise plus balanced diet for seven consecutive days significantly improved whole body and peripheral insulin sensitivity by $37.5 \%(\mathrm{p}<0.05)$ and $44.4 \%(\mathrm{p}<0.0001)$, respectively. These variables were unchanged in the balanced diet only group [16]. The remaining two studies reported no change in homeostasis model assessment for insulin resistance (HOMA-IR) and quantitative insulin check index (QUICKI) following four and twelve weeks of exercise training $[14,15]$.

Glucose control: All three studies reported no difference in fasting glucose levels across groups following 7-d, four and twelve weeks of exercise training [14-16]. However, Jorge et al reported that within group comparisons showed that aerobic, resistance and combined aerobic plus resistance exercise training for twelve weeks significantly $(\mathrm{p}<0.05)$ lowered fasting glucose by $15.6 \%, 17.0 \%$ and $9.0 \%$, respectively [15].

Blood lipids: Two studies assessed lipid profile. Of these, one study reported positive findings; Hordern et al. [19] reported that when compared with usual care, four weeks of exercise training produced a small but significant decrease in TG; $0.2 \%(\mathrm{p}<0.003)$. By contrast, Jorge et al [20] reported no change in TG across groups following twelve weeks of exercise training, although within group comparisons showed that aerobic, resistance and combined aerobic plus resistance exercise training for twelve weeks significantly $(\mathrm{p}<0.05)$ decreased TG by $11.2 \%, 52.9 \%$ and $19.8 \%$, respectively.

Inflammation: One trial examined inflammatory markers. While there was no statistical difference in high sensitivity C-reactive protein (hs-CRP) across groups, within group comparisons indicated that twelve weeks of aerobic, resistance and combined aerobic and resistance exercise training for twelve weeks significantly $(\mathrm{p}<0.05)$ decreased hs-CRP by $10.8 \%, 15.0 \%$ and $10.6 \%$, respectively [15].

Anthropometry: All three studies included in the current review described anthropometric data and reported that when compared with a no exercise control group, exercise training had small or no significant effect on body composition including body weight (BW), body mass index (BMI) body fat (BF).

Aerobic fitness: Three studies reported on aerobic fitness. Hordern et al reported small but significant increase in maximal aerobic capacity $\left(\mathrm{VO}_{2} \mathrm{max}\right)$ of $1.4 \%(\mathrm{p}<0.011)$ following four weeks of exercise training [14] while the remaining two reported no change $[15,16]$. Jorge et al however, reported that although there was no significant difference across the groups, within groups comparison showed that aerobic exercise training for twelve weeks significantly increased peak oxygen consumption $\left(\mathrm{VO}_{2}\right.$ peak) by $14.0 \%(\mathrm{p}<0.05)$, while aerobic and/ resistance training did not [15].

\section{Discussion}

The current review demonstrates that short-term exercise improves insulin sensitivity and glucose control in overweight/obese adults without changes in body composition and/aerobic fitness. Thus, shortterm exercise may be applicable as the first line of treatment for insulin resistance and type 2 diabetes. Short-term exercise interventions offer opportunity for close supervision which has been associated with better adherence to exercise programs. Furthermore, the improvements observed with short-term exercise training may encourage individuals to adopt a physically active lifestyle [17].

The current review shows that exercise training for seven consecutive days increased whole body insulin sensitivity, reported in the study by Winnick et al [16] while longer duration studies of four and twelve weeks reported no change $[14,15]$. The differences could be attributed to higher intensity exercise prescription in the study by Winnick et al [16]. Previous studies have shown that when compared with moderate intensity training, higher intensity training produced superior benefits in insulin sensitivity $[18,19]$. Recent reports have shown that during high intensity training there is recruitment of a larger proportion of muscle fibres and greater number of type 2 glycolytic muscle fibres, adaptations which contribute to glycogen depletion-induced insulin sensitivity [20,21]. Also, the study by Winnick et al used direct measures of insulin sensitivity while the other studies used surrogate markers (HOMA indices) [14,15]. Indices of insulin resistance have been found to correlate well with direct measures such as the hyperinsulinemic clamp but are not necessarily equivalent [22].

This review also shows that short-term exercise improves triglycerides even at moderate intensity. This is in agreement with a previous review which reported that exercise improves dyslipidaemia in physically inactive individuals [23]. The mechanism by which exercise improves dyslipidaemia remains unclear. Studies however, have suggested that this could be due to exercise-induced activation of lipoprotein lipase (LPL) which is responsible for the hydrolysis of triglycerides [24]. Lipoprotein lipase can be active for up to 24 hours following one hour of exercise [25]. Thus, short-term exercise may be effective for instantaneous improvements in triglycerides which would avertedly improve the prognosis cardiovascular disorders associated with insulin resistance [26].

Studies in this review have shown that short-term exercise does not result in measurable changes in body composition and aerobic fitness. However, aerobic training was found to produce favourable effects on cardiorespiratory fitness compared with resistance training $[14,15]$. This was in contrast with previous review and meta-analysis which reported that resistance training improved cardiorespiratory fitness in a similar manner as aerobic training [27].

This review contributes to information for future research in understanding effects short-term exercise protocols on metabolic disorders associated with overweight/obesity in physically inactive adults. Our findings are to be interpreted with caution however, because the current review describes a small sample size and studies published in English only.

In the current review, only three studies met the inclusion criteria, suggesting that despite evidence of benefit of short-term exercise, randomised controlled trials are lacking. Trials included in this review were mostly on older adults, and the exercise prescriptions were moderate intensity. Since diabetes is increasingly affecting younger people and exercise training at high intensity is increasingly reported 
to produce superior improvements on metabolic parameters; with half the time required for moderate intensity training, future randomized controlled trials to investigate short-term effects of high intensity exercise in younger insulin resistance/diabetes patients are warranted.

In conclusion, the current review provides useful information on the possible clinical applications of short-term exercise to improve insulin sensitivity and control glycaemia. Findings of the current review also show that the therapeutic effects of exercise can occur without changes in body composition and aerobic fitness. Therefore, in addition to encouraging changes in body composition of overweight/ obese patients through exercise, improvements in metabolic health which precede changes in body composition should be emphasized.

\section{Funding}

The authors would like to thank the University of KwaZulu-Natal College Of Health Sciences (CHS) and National Research Foundation (NRF) for funding received.

\section{References}

1. Jung U, Choi MS (2014) Obesity and its metabolic complications: The role of adipokines and the relationship between obesity, inflammation, insulin resistance, dyslipidemia and nonalcoholic fatty liver disease. Int J Mol Sci 15: 6184-6223.

2. Patel TP, Rawal K, Bagchi AK, Akolkar G, Bernardes N, et al. (2016) Insulin resistance: An additional risk factor in the pathogenesis of cardiovascular disease in type 2 diabetes. Heart Fail Rev 21: 11-23.

3. Zhang P, Gregg E (2017) Global economic burden of diabetes and its implications. Lancet Diabetes Endocrinol 5: 404-405.

4. Goossens GH (2008) The role of adipose tissue dysfunction in the pathogenesis of obesity-related insulin resistance. Physiol Behav 94: 206-218.

5. Way KL, Hackett DA, Baker MK, Johnson NA (2016) The effect of regular exercise on insulin sensitivity in type 2 diabetes mellitus: A Systematic review and meta-analysis. Diabetes Metab J 40: 253-271.

6. Naufahu J, Elliott B, Markiv A, Dunning-Foreman P, McGrady M, et al. (2017) High intensity exercise decreases ip6k1 muscle content \&amp; improves insulin sensitivity $\left(\mathrm{si} 2^{*}\right)$ in glucose intolerant individuals. J Clin Endocrinol Metab 103: 1479-1490.

7. Francois ME, Durrer C, Little J (2017) High-intensity interval training with or without post-exercise milk consumption improves cardiovascular function in patients with type 2 diabetes. The FASEB Journal 31: 1035.

8. Siddiqui N, Nessa A, Hossain M (2010) Regular physical exercise: Way to healthy life. Mymensingh Med J 19: 154-158.

9. Moher D, Shamseer L, Clarke M, Ghersi D, Liberati A, et al. (2015) Preferred reporting items for systematic review and meta-analysis protocols (prisma-p) 2015 statement. Syst Rev 4: 1.

10. Regensteiner JG, Shetterly SM, Mayer EJ, Eckel RH, Haskell WL, et al (1995) Relationship between habitual physical activity and insulin area among individuals with impaired glucose tolerance: The san luis valley diabetes study. Diabetes Care 18: 490-497.

11. Summers L, Fielding B, Bradshaw H, Ilic V, Beysen C, et al. (2002) Substituting dietary saturated fat with polyunsaturated fat changes abdominal fat distribution and improves insulin sensitivity. Diabetologia 45: 369-377.

12. Bell LM, Watts K, Siafarikas A, Thompson A, Ratnam N, et al. (2007) Exercise alone reduces insulin resistance in obese children independently of changes in body composition. The J Clin Endocrinol Metab 92: 4230-4235.

13. Porritt K, Gomersall J, Lockwood C (2014) Jbi's systematic reviews: Study selection and critical appraisal. Am J Nurs 114: 47-52.

14. Hordern MD, Cooney LM, Beller EM, Prins JB, Marwick TH, et al. (2008) Determinants of changes in blood glucose response to short-term exercise training in patients with type 2 diabetes. Clin Sci (Lond) 115: 273-281.

15. Jorge ML, de Oliveira VN, Resende NM, Paraiso LF, Calixto A, et al. (2011) The effects of aerobic, resistance, and combined exercise on metabolic control, inflammatory markers, adipocytokines, and muscle insulin signaling in patients with type 2 diabetes mellitus. Metabolism 60: 1244-1252.

16. Winnick JJ, Sherman WM, Habash DL, Stout MB, Failla ML, et al. (2008) Short-term aerobic exercise training in obese humans with type 2 diabetes mellitus improves whole-body insulin sensitivity through gains in peripheral, not hepatic insulin sensitivity. J Clin Endocrinol Metab 93: 771-778.

17. Resnik M, Galvani C, Sartorio A (2003) Effects of non-specific vs individualized exercise training protocols on aerobic, anaerobic and strength performance in severely obese subjects during a short-term body mass reduction program. J Endocrinol Invest 26: 197-205.

18. Cockcroft EJ, Williams CA, Tomlinson OW, Vlachopoulos D, Jackman SR, et al. (2015) High intensity interval exercise is an effective alternative to moderate intensity exercise for improving glucose tolerance and insulin sensitivity in adolescent boys. J Sci Med Sport 18: 720-724.

19. Ross R, Hudson R, Stotz PJ, Lam M (2015) Effects of exercise amount and intensity on abdominal obesity and glucose tolerance in obese adults: A randomized trial. Ann Intern Med 162: 325-334.

20. Scribbans TD, Edgett BA, Vorobej K, Mitchell AS, Joanisse SD, et al (2014) Fibre-specific responses to endurance and low volume high intensity interval training: Striking similarities in acute and chronic adaptation. PLOS ONE 9: e98119.

21. Prior SJ, Goldberg AP, Ortmeyer HK, Chin ER, Chen D, et al. (2015) Increased skeletal muscle capillarization independently enhances insulin sensitivity in older adults after exercise training and detraining. Diabetes 64: 3386-3395.

22. Abbasi F, Silvers A, Viren J, Reaven GM (2018) Relationship between several surrogate estimates of insulin resistance and a direct measure of insulin-mediated glucose disposal: Comparison of fasting versus postglucose load measurements. Diabetes Res Clin Pract 136: 108-115.

23. Wang Y, Xu D (2017) Effects of aerobic exercise on lipids and lipoproteins. Lipids Health Dis 16: 132.

24. Ghafouri K, Cooney J, Bedford DK, Wilson J, Caslake MJ, et al. (2015) Moderate exercise increases affinity of large very low-density lipoproteins for hydrolysis by lipoprotein lipase. J Clin Endocrinol Metab 100: 2205-2213.

25. Ferguson MA, Alderson NL, Trost SG, Essig DA, Burke JR, et al. (1998) Effects of four different single exercise sessions on lipids, lipoproteins, and lipoprotein lipase. J Appl Physiol 85: 1169-1174.

26. Jorgensen AB, Frikke-Schmidt R, Nordestgaard BG, Tybjaerg-Hansen A (2014) Loss-of-function mutations in apoc3 and risk of ischemic vascular disease. N Engl J Med 371: 32-41.

27. Matthew H, Yorgi M, Jonathan F, Fiatarone SM (2017) The effect of progressive resistance training on aerobic fitness and strength in adults with coronary heart disease: A systematic review and meta-analysis of randomised controlled trials. Eur J Prev Cardiol 24: 1242-1259. 